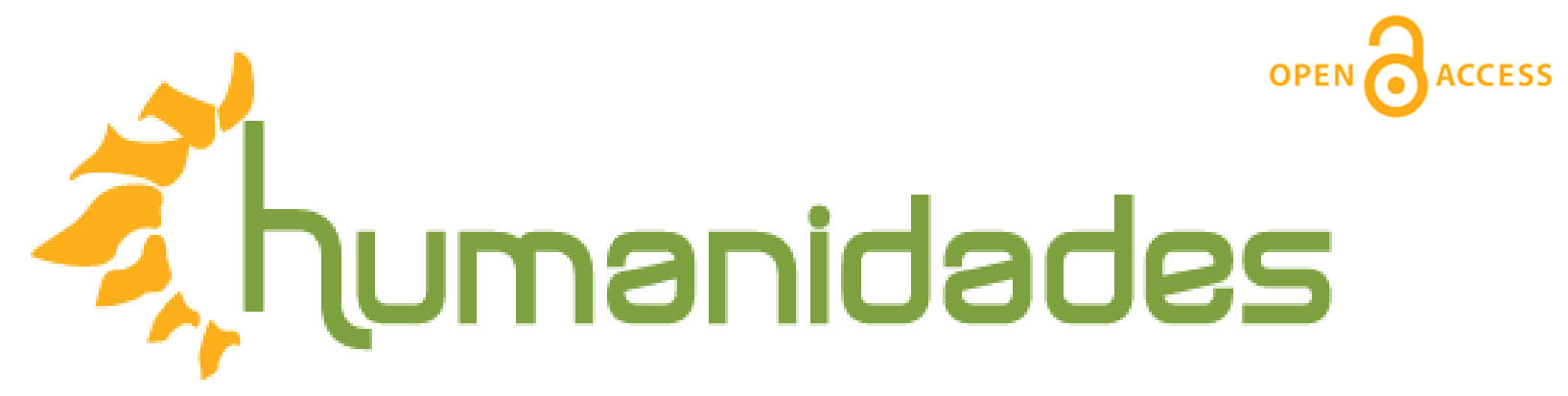

Revista de la Escuela de Estudios Generales, Universidad de Costa Rica

Enero-junio, 2017 • Volumen 7, número 1 • EISSN 2215-3934•pp. 1-24

Recibido: 12-Febrero-2016 Aceptado: 18-Mayo-2016

\title{
Las intimidades colectivas de la clase obrera. Vida urbana y cultura popular en las ciudades de principios del siglo $\mathrm{XX}$
}

DOI: http://dx.doi.org/10.15517/h.v7i1.27622

\section{Francesc Calvo Ortega}

Docente en la Universidad de Almería, España. Director del Programa de Mayores y del Seminario de Humanidades y Ciencias de la Información, en dicho centro educativo.

Correo electrónico: fracalvo@ual.es

Todos los derechos reservados. Universidad de Costa Rica. Esta revista se encuentra licenciada con Creative Commons. Reconocimiento-NoComercial-SinObraDerivada 3.0 Costa Rica.

Correo electrónico: humanidades@ucr.ac.cr / Sitio web: http://revistas.ucr.ac.cr/index.php/humanidades 


\title{
Las intimidades colectivas de la clase obrera. Vida urbana y cultura popular en las ciudades de principios del siglo XX
}

\section{Resumen}

A menudo se habla en referencia a la clase obrera de una cultura de resistencia traducida en procesos de reapropiación. Una cosa es cierta: de una forma o de otra, el obrero reelabora con otras finalidades las estructuras y las normas sociales que le son impuestas. Pero el paso al acto de autonomía como viene a significar esa reapropiación está en función de los recursos de que dispone y varía según la conciencia individual, el grupo familiar o la organización política de la cual el individuo puede formar parte. Se hace patente, pues, un nivel de autodeterminación, como margen para la libertad individual, que permitiría una tipo de "cambio interior" y que haría posible a cada cual resistir las normas y regular la intensidad y la combinación entre el ámbito de lo colectivo y el ámbito de lo privado. La moral colectiva se construye en este entorno en que el imaginario social articula todo un entramado de representaciones autónomas.

\author{
Palabras claves: Cultura \\ popular, clase obrera, \\ sociología urbana, \\ planificación urbana, política \\ de la vivienda.
}

\section{The Collective Intimacies of the Working Class. Urban Life and Popular Culture in Cities of the Beginning of the XXth Century}

\begin{abstract}
Often one speaks in reference to the working class of a culture of resistance translated in processes of reappropriation. A thing is true: of a form or of other one, the worker re-prepares with other purposes the structures and the social norms that are imposed on him. But the step to the autonomy act since it comes to mean this reappropriation is according to the resources which he has and changes according to the individual conscience, the familiar group or the political organization of which the individual can be part. There becomes clear, then, a level of selfdetermination, like margin for the individual freedom, which would allow a type of "interior change" and which there would make possible to resist the norms to everyone and to regulate the intensity and the combination between the ambience of the collective field and the ambience of the private field. The collective morality is constructed in this environment in which the imaginary social one articulates the whole framework of autonomous representations.
\end{abstract}

Keywords: Popular Culture, working class, urban sociology, urban planning, housing policy. 
Explicar el proceso de identificación de los obreros con el trabajo, el entorno o la cultura por medio de un análisis puramente clasista o de cariz económico resulta insuficiente en el marco analítico de una historia sociocultural del movimiento obrero. Los comportamientos colectivos e individuales, en la fábrica, la taberna o el café, con la familia, las reuniones de grupos con afinidades deportivas, literarias, musicales, o incluso gastronómicas, contienen una significación política que atraviesa la vida cotidiana de los miembros de la clase trabajadora. Asimismo, si intentáramos demostrar el desarrollo y la estructura de la proletarización de los campesinos se volvería insuficiente si lo explicásemos centrándonos solamente en su arraigo a los valores rurales y a su comunidad de origen. La mayoría de estudios sobre la vida cotidiana han tenido que hacer frente a la misma problemática: las relaciones complejas que se entrelazan en el interior de las condiciones materiales, económicas y sociales de la vida diaria, en la forma en que los esquemas de comportamiento específicos se estructuran, es decir, cómo los grupos de personas se reproducen material, social y culturalmente.

En la autoproducción del pasado y la imagen que tienen de ellos mismos, los obreros no están aislados. El medio familiar, el medio local, el barrio, el pueblo y la ciudad, contribuyen a la producción de la propia imagen donde se valoriza el trabajo industrial y a todos aquellos que están destinados a realizarlo. La fábrica y el mundo cotidiano que la rodea no parecen estar sistemáticamente excluidos de la vida fuera del trabajo. Tal como nos explica Catherine Paradeise (2003), no es difícil comprobar en un contexto de 
paternalismo empresarial, la existencia de elementos de interpenetración entre el mundo del trabajo y el tiempo de ocio. Por ejemplo, la actitud de las esposas de los obreros metalúrgicos es bastante reveladora: siempre que se ha tenido acceso a sus testimonios, comprobamos que intervienen sin titubear para añadir alguna precisión técnica o apreciación sobre las condiciones de trabajo.

Muchos lugares de la geografía industrial de los siglos XIX y XX, la ciudad y la fábrica están en permanente simbiosis, no únicamente por la naturaleza del entramado urbano sino también por la red social de obreros, de una estructura entrelazada de relaciones familiares y de amistad que unen a los trabajadores de las mismas fábricas o de empresas vecinas (cf. Segrestin, 1980). Las prácticas de autoproducción del movimiento obrero son inseparables de los procesos de acumulación de un cierto "patrimonio" social, económico y cultural. Este patrimonio, acumulado de generación en generación, es constitutivo de la identidad social local y por lo tanto también lo es de la construcción identitaria de los obreros.

Las prácticas de la cultura popular se desarrollan bastante a menudo en un espacio caracterizado por la proximidad del domicilio. El espacio social de la clase obrera está en función de esta territorialidad y a partir de ahí se pueden explicar los usos característicos de su cultura y la diversidad que subyace. La vivienda representa uno de los principales elementos que configuran las prácticas dadas en la cotidianidad material de las clases sociales (Magri, 1997). En la parte inferior de la jerarquía social encontramos la "vivienda barata" —que los nuevos modelos de construcción denominan "social"-, y 
no es nada superficial o inútil conocer su fisonomía a pesar de que el cuadro de posibilidades arquitectónicas es limitado. Pero sería erróneo reducir al horizonte de una homogeneidad constructiva de las viviendas el espacio doméstico de la población que subsiste del trabajo industrial. Las experiencias residenciales divergen y se expresan por medio de las formas de reapropiación del alojamiento, es decir el "consumir" en un sentido práctico del término. Michel de Certeau (1980) explica que a una producción racionalizada, expansionista y la vez centralizadora, le corresponde otra producción que califica de «consumación» cultural. Se trata de una práctica intrincada y dispersa pero que se insinúa por todas partes, más bien silenciosa y nada espectacular pues no aparece como el producto impuesto por un orden económico dominante sino en la forma de utilizarlo.

Anulando el vínculo monetario entre producto y consumidor, los actos de provecho material de los objetos toman sentido a través de las estrategias de interpretación que construyen sus significaciones. Así, para conocer las formas populares del habitar no basta con el inventario y descripción de todos aquellos objetos que pueblan el interior del inmueble, sino que hay que observar su presentación, la manera en que están dispuestos y la importancia que tienen para los que allí viven en la escena doméstica como una apropiación diferenciada según la clase social. Aun así, no existe una discontinuidad absoluta entre clases. Hemos de admitir que las formas de habitar en el grado inferior de las jerarquías no ignoran los elementos que forman parte del inmueble burgués pero lo hacen a partir de un proceso que 
reelabora los usos iniciales y una vez reapropiados se convierten en productos interiores diferenciados.

El gusto popular no destierra, ignorándolas, todas aquellas normas y exclusiones en la forma de habitar de las clases dominantes. Estrechamente informadas por los trabajadores domésticos, las clases populares incorporan ciertas prácticas de la cultura burguesa bajo una forma particular de clase, donde se combina un sistema de proscripción moral ligado a la condición social de la burguesía y un abanico de recursos culturales que responden tanto en su origen como en la posición a la trayectoria social de la clase obrera. Comparada con la amplitud cómoda de un apartamento burgués, la disposición y distribución de un espacio sencillo manifiesta sin ningún género de duda la desigualdad existente entre las clases sociales. A pesar de ser observada como una variante de las prácticas populares, una mirada atenta — más al detalle de aquello que se nos muestra como el valor de los objetos-, en algunos casos, la vivienda nos indica una forma de dominación cultural y la materialización simbólica de una voluntad de ascenso social, signo inequívoco de la evolución económica de las profesiones intermedias entre el proletariado y la clase dirigente (Barbey, 1990). Aunque hay algo más que esto.

Llevar a cabo un ejercicio histórico de contrastación entre los modelos banalizados de la vivienda social de principios del siglo XX — como espacio de experimentación moral del urbanismo reformador-, con las aspiraciones y deseos populares en materia de alojamiento, nos obliga a considerar la existencia de modelos culturales diferenciados en la sociedad urbana en la 
quedan desplegadas formas de vida expresadas por los modos de reapropiación residencial de los espacios destinados a vivir bajo un techo (Faure, 1999). La forma en que se emplea la vivienda como producto impuesto para vivir en la ciudad, denota una cultura del espacio propia de los moradores en relación a las costumbres burguesas y aristocráticas de habitar sus propiedades. Más allá de los resultados de una comparación entre rangos sociales distinguidos por una profunda desigualdad económica, que bien sabemos existía, una observación más al detalle de esta diferencia entre un hogar espacioso y de ostentación y un espacio modesto y reducido - a veces se trata de un simple habitáculo_-, nos indica en cambio ciertas similitudes en la disposición mínima de organizar un hogar entre las diferentes clases sociales. Asimismo, por una especie de trasposición correlativa entre diversas categorías de obreros, los modos de influencia en la transformación del hábitat entre los trabajadores cualificados, los oficiales de los talleres artesanos y el proletariado, como variantes diferenciadas de las prácticas populares, se explican a través de una materialización simbólica que alude a los deseos de una mejora profesional.

El estudio del mobiliario conduce a conclusiones parecidas (Magri, 1997). Para el inmigrante, o el joven obrero de provincias, recién llegado a la ciudad, la única opción está en alquilar una habitación pensionada, y al menos, durante un tiempo y de forma regular, habita diversos lugares cuyo menaje existe en función del tránsito regular de personas que como él ocupan temporalmente ese espacio. Para el obrero que lleva tiempo instalado en la 
ciudad tener sus "propios" muebles es una necesidad. Y por ello hace lo posible por acceder a los mercadillos o los almacenes de venta a crédito, de clientela más bien popular. Aquí la posesión poco tiene que ver con las aspiraciones de la propiedad burguesa, sentimiento de una clase ajena a las formas de vida populares. Poseer un mobiliario, aunque frugal y muchas veces humilde, no deja de ser un gesto de independencia y asentamiento en una forma de existencia determinada, de disfrutar de los objetos que se disponen en la vivienda, intercambiarlos y, si las cosas van mal, venderlos (Faure, 1998).

La voluntad de permanencia está cada vez menos condicionada por el mercado de alquiler que por las necesidades de espacio y la capacidad económica para poder satisfacerlas. Las familias son cada vez más numerosas y ciertas exigencias arrendatarias - muchos propietarios son renuentes a alquilar a las familias cargadas de hijos-, dificultan las posibilidades de encontrar una vivienda en condiciones. En estas circunstancias, las formas de habitar el espacio son bastante representativas de la incomodidad propia de la vivienda popular. Lo que por el día funciona de comedor por la noche se dispone como dormitorio, un espacio donde el uso de las camas se distribuye evitando en lo posible la promiscuidad, hijos de distinto sexo y edad, entre padres e hijos o por la súbita presencia de un amigo o familiar que ha llegado a la ciudad — de ahí la innovación que supondrá a finales del siglo XIX la invención del sofá-cama para las familias con poco margen de maniobra para superar la escasez de espacio- 
No se trata tanto de una moral, que en la mayoría de los casos viene impuesta desde el exterior $-y$ que se aprovecha circunstancialmente de la "miserabilidad" para imponerla al estilo de una pedagogía—, sino de unas costumbres de la penuria y la precariedad que determinan durante largo tiempo una cultura de la vida privada y doméstica de los obreros. Entre estas dos formas que estructuran la vida en el hogar obrero, una ética de las clases populares - que no obtiene su punto de anclaje en la miseria sino en la humillación de una existencia pobre y, a su vez, en la aspiración de llegar a conquistar un lugar donde residir en condiciones-, encuentra en la vivienda social aquel espacio que aún asimilando un nuevo modelo de vivir la ciudad no las desposeen de su cultura ni de su afán de resistencia a una probable racionalización de sus costumbres.

Toda una historia del medio urbano constata la existencia de una comunidad diferenciada en la forma de habitar expresando a la vez la rápida circulación de los modelos de consumición que afecta directamente a las preferencias originales de las clases populares (Harloe, 1995; Cohen y Baudouï, 1995). Los testimonios de principios del siglo XX expresan la rápida asimilación de una cultura ciudadana alejada de la cultura rural. La instalación del menaje de origen popular en la ciudad viene acompañada de una aculturación que transforma las preferencias y las formas de presentar el hábitat a los demás. Esta asimilación de nuevos modelos domésticos por parte de las clases populares nos indica que no se trata ni de un desfallecimiento cultural ni de una reluctancia hacia la sociabilidad tradicional que hasta entonces daba 
sentido a formas de vida no urbanas (cf. Murard y Zylberman, 1976). En todo caso, manifiesta un registro contrario a un esquema de convenciones uniformadoras, inducidas por el estilo de vida de las clases altas: las formas de sociabilidad popular no están calcadas a las fijadas por el mundo burgués, porque ni los hábitos, anteriores al arrendamiento, de una forma de ser habitante quedan anuladas, ni la recomposición identitaria del obrero en la ciudad rompe con la voluntad de afirmar una dignidad de clase en la cual se siente integrado.

Los beneficiarios de las viviendas "baratas" no lo son en función de su pertenencia a una clase trabajadora empobrecida, sino por la falta de recursos para abandonar unos espacios malsanos y conflictivos (Magri, 1991). Es la peligrosidad sobrevenida de las personas que carecen de medios materiales y no la clase social aquello que define una población de beneficiarios de la política de la vivienda (Warin, 1995). Esta política se lleva a cabo con un carácter preventivo y por tanto se abandonan las estrategias caritativas, ordinariamente puntuales, por una gestión de la pobreza a largo plazo en la que media una proliferación de viviendas sociales como enclaves para una guerra contra la miseria tanto física como moral de las clases trabajadoras.

Una nueva visión política y administrativa del movimiento obrero, desde arriba y profundamente liberal - y que es producto de esa orientación reformadora del modo en que las clases populares habitan el espacio urbano-, establece un nuevo estatuto pacificador de las voluntades 
revolucionarias que en Francia tuvieron como punto de arranque la Comuna de París de 1830 (cf. Donzelot, 2007). Por un lado, la Ley, a propuesta de las instituciones de reforma, eleva al obrero al rango de "ciudadano" no reconociéndole otro estatus que el de "particular" y, por otro, le niega una identidad de clase silenciando cualquier mención acerca de su de pertenencia a un movimiento o clase. De ahí que toda táctica o estrategia que involucra a los obreros en las redes asistenciales de la ciudad diluya un reconocimiento explícito a un colectivo y los confunda en el interior de una amalgama irreconocible de individuos pobres que desean acceder a un techo a través de la vivienda barata. Entonces la Ley no los reconoce sino como "individuos", el condicionante básico para que sean tratados como ciudadanos, igualando derechos pero manteniendo las desigualdades.

Denegación simbólica de la división de clases sociales que establece, en un gesto institucional de reducción de lo colectivo emergente, las bases constitutivas de una política social desagregada del mundo del trabajo, - - y todo lo que implica la negación de una existencia sociocultural del movimiento obrero-, entrando así de lleno en el ámbito de las políticas urbanas que actúan sin distinciones frente a la miseria cuyo origen, fundamentalmente, es la falta de trabajo o la precariedad de este.

El despliegue de las tácticas "familiaristas" de los reformadores urbanos de principios de siglo, que ven en el concubinato los resortes específicos de una "civilización popular" como tributo a una renovación de las "clases 
peligrosas" en la ciudad, de las que ya había hablado Louis Chevalier (1984), es el signo y soporte de una reorganización "administrativa" de la vida privada de la clase trabajadora. Los obreros no defienden el concubinato a la manera de un hábito popular, representativo de un modo de vivir las relaciones entre los dos sexos. Más bien es el escaso interés por parte del hombre de contraer matrimonio, lo que está detrás de esta práctica que se extiende en coexistencia con las relaciones ilegítimas entre una mujer de clase baja y un burgués (Battagliola, 1995). De ahí que las estrategias matrimoniales no puedan disociarse de las estrategias sociales tanto de la clase burguesa dominante -como táctica para extender una moral de rigor-, como para la mujer de las clases populares — con el objetivo de alcanzar una estabilidad y una movilidad social que, aunque de poco recorrido, le garantice una seguridad de la que no gozan las solteras- (cf. Farge y Klapish-Zuber, 1984).

Con todo, el punto de partida de la mujer que desea el matrimonio preferencia ante la posibilidad de una serie de rupturas que la "deslocaliza" de los barrios a los que sucesivamente se desplaza como concubina-, conforma un itinerario de fragilidad en cuanto al juego de las seducciones, $\mathrm{y}$ al peligro del abandono por parte del marido, poco dado a las formalidades de este tipo, de acabar devaluada moralmente en ese mercado difícil de los enlaces conyugales y bajo el control estricto de las instituciones sociales que distribuyen las ayudas, o en última instancia condenada a la práctica de relaciones ilícitas (Fairchild, 1978). Asimismo, las astucias que genera un deseo de movilidad social, siempre en función de lograr casarse con algún 
miembro de la pequeña burguesía, conduce a las jóvenes obreras a aceptar vivir en concubinato como paso previo a un matrimonio como el único medio de escapar a su condición social.

Según se ha desarrollado históricamente, teniendo en cuenta además la evolución de las estructuras sociales, el concubinato aparece como una práctica con diferentes perspectivas (Battagliola, 1995). Ocupa un lugar entre el amor y lo venal, a veces quizá una mezcla de los dos, y el matrimonio, que más allá de representar una aspiración individual, permanece regularmente subordinado al acceso a una posición social. A partir de principios del siglo $\mathrm{XX}$ las uniones estables ganan terreno en relación al concubinato estableciendo una nueva tendencia en relación a la segunda mitad del siglo XIX. Lo reflejan tanto el aumento de aquellos padres que reconocen a los hijos fuera del matrimonio como la proliferación de ayudas concedidas por parte de las instituciones que lo promocionan. A esto habría que añadir la legalización del divorcio en algunos países de Europa, que permite revocar lo que aparece como un contrato entre dos personas, sin más. (Léridon y Villeneuve-Gokalp, 1994). En el centro del debate político lo que está en juego es la dignidad de la mujer y la protección a la infancia. Más allá de la crítica y la lucha a favor o en contra, para las clases populares el matrimonio contrabalancea entre dos polos: la desvalorización del mismo como unión sagrada e indisoluble y su utilidad como herramienta útil para superar los filtros morales de la Reforma Social, en vías de instaurar el matrimonio a modo de una célula restringida (reducida y localizada) en la que se van a 
intensificar los elementos fundamentales y las relaciones propias de la familia contemporánea, tal y como hoy la conocemos (cf. Boucher, 2011).

Recuperar una perspectiva de observación a pequeña escala sobre la cotidianidad de las clases trabajadoras, nos permite tener en cuenta aspectos

La vida en el barrio esenciales de la vida urbana y comprender así una realidad aproximada de la existencia popular en este medio, especialmente en épocas en que la proximidad juega un papel fundamental en las relaciones sociales y la organización de la vida comunitaria (Faure, 1993). Observando de cerca al obrero en la ciudad, los desplazamientos que realiza para ir a la fábrica y la movilidad posterior cuando acaba la jornada trabajo, se descubre que la proximidad más inmediata, la distancia corta, representa la medida más habitual de las idas y venidas.

El trabajo a domicilio, muy frecuente en el cambio de siglo, también reduce la amplitud del radio de desplazamientos básicos que estructuran la existencia de las clases populares alrededor de su espacio vital de sociabilidad, el barrio. Medio popular por excelencia, el barrio se presenta como el marco casi obligado donde acontecen toda una serie de acciones íntimas y colectivas que dan lugar a un conocimiento comunitario: por ejemplo, la discusión sobre la preparación de las comidas, el intercambio de enseres de cocina entre el vecinos, la alimentación y el cuidado de los niños cuando sus progenitores están trabajando, o el sistema de crédito sin intereses empleado por los tenderos. El barrio no deja de ser la expresión de un medio local donde la influencia sobre las existencias de sus habitantes tiene una medida concreta. 
Es decir, el lugar que cada uno conoce y frecuenta más que otro, como el entorno residencial de recognición de la propia realidad individual y colectiva. Individual porque cada cual vive en el interior de una red social de relaciones que no es la misma que la de sus vecinos, por cuestiones de sexo, edad, trabajo, militancia, etc., y colectiva pues el espacio está investido por la vida comunitaria local que resume la familiaridad de los contactos entre un individuo y los demás. Una villa popular, con este nombre se denomina el barrio de Gràcia en Barcelona, no deja de ser una forma de decir que todo el mundo, con frecuencias diferenciadas, se conoce, que las posibilidades de verse y hablar se producen a menudo, y por lo tanto se erige como el espacio de vida y evolución social que la comunidad acaba reconociendo como la propia.

Sin embargo, afirmar que la cultura popular en los barrios se articula alrededor de la idea de que cada cual conoce la vida de su vecino es una forma un poco desviada de indagar una vida comunitaria. Las conversaciones de ventana a ventana, las discusiones familiares en público, los chismes que circulan por los bloques de viviendas, las miradas y las escuchas indiscretas, todo esto existe y se da por hecho aunque debe ser correctamente interpretado y ubicado en el contexto de los valores y comportamientos que las clases populares practican (Faure, 1994). La distinción entre vida pública y vida privada, entre conocimiento del otro e intimidad, se comprende a través de una moral colectiva que establece unos límites bastante precisos de la familiaridad en el barrio, de cuándo es preciso actuar de forma individual o colectiva y cuándo no se debe intervenir ante un suceso determinado. 
Las existencias individuales no obedecen a una regla homogénea del vivir en el barrio, pues ni los rangos sociales, ni los profesionales, y menos aún los culturales, son los mismos. Una pequeña burguesía local está presente incluso en el barrio más pobre. Esto es cierto en la medida en que los pequeños comerciantes y los pequeños propietarios están integrados, y apenas se distinguen por su estatus y un modo de actuar que asume los valores de rechazo general a los intermediarios, los usureros, los acaparadores, etc. De la misma forma se impugna el intrusismo institucional o administrativo: la policía, un oficial del departamento de justicia, ¿acaso se presentan en el barrio a velar por el cumplimiento de las obligaciones por parte de todos, a garantizar la seguridad de sus habitantes?

Las sociedades de caridad también han conocido las dificultades para "entrar" en los barrios populares con la intención de resolver los problemas de la comunidad. Si se trata de individuos con finalidades de exploración, periodistas, encuestadores, investigadores, la reacción también puede ser agresiva: aún hoy se dan las circunstancias en las que difícilmente alguien desconocido pueda esperar una cordial bienvenida en un barrio popular (Dollé, 1990). ¿Se recluye entonces el barrio sobre sí mismo, dejando en los límites territoriales del mismo un trato con el mundo exterior? El contacto de lo externo en el interior del barrio genera suspicacias, y más aún si se trata de instituciones administrativas del Estado: si alguien es requierido por algún organismo en todo caso hay que trasladarse donde éste tiene sus oficinas y si no, como ocurre con cierta frecuencia, mejor ignorarlo y desaparecer en el 
viscoso magma del barrio que sólo admite presencias por invitación. El barrio protege contra las apariciones indiscretas.

Si el barrio se establece como una barrera hacia el mundo oficial de la administración, de las Sociedades filantrópicas de ayuda al pobre o los encuestadores sobre el trabajo a domicilio, uno de los focos principales de atención se centra alrededor del problema de la salud y la higiene de las familias obreras (Dean, 1991). El discurso higienista iniciado a mediados del siglo XIX parte de una actividad autónoma frente a la medicina de la época, diferenciándose en el nivel de intervención: el higienismo, no es la etiología de las posibles patologías orgánicas de la población sino que es su frecuencia y su difusión diferencial lo que le interesa, es decir, en su objeto principal de intervención está el conjunto de características que provocan la insalubridad en el alojamiento popular y su extensión en el cuerpo social (cf. Frioux, Fournier y Chauveau, 2011). Y todavía más.

El higienismo interviene allí donde la medicina, en un primer momento, no puede hacerlo. Entre el medio urbano y los cuerpos el higienismo intenta relacionar la actitud del individuo frente a la enfermedad, la salud y la habitabilidad: trata de hacer emerger la conducta desviada del individuo a partir de una relación de causa-efecto — por ejemplo, que la insalubridad de muchos inmuebles es la causante de ciertas conductas "patológicas", alcoholismo, delincuencia, prostitución, absentismo laboral...-. El higienismo es el instrumento para hacer coincidir frecuencias patológicas y clases populares a través de una interposición moral. 
La inculcación de hábitos de higiene en las clases populares tienen en este entrecruzamiento su origen, el momento en que el individuo contrae sus deberes morales y cívicos en relación a su salud, la de su familia y la de la sociedad en su conjunto. La salud adquiere de esta forma, además de una dimensión pública, una dimensión política, siempre alrededor de esa atención dirigida por parte del poder político-administrativo a las formas de vida de las clases populares y a la necesidad de reformarlas (Murard y Zylbermann, 1986). Con esto queremos decir que el dominio político-médico de las poblaciones no sólo concierne a prescripciones referentes a la enfermedad sino también a las formas generales de la existencia y del comportamiento social (alimentación, sexualidad, trabajo y habitabilidad).

Las ambiciones del higienismo abren para la medicina, a finales del siglo XIX, una era floreciente de inspecciones, encuestas y estadísticas, con el objetivo de fijar, contabilizar y corregir la miseria como sus efectos derivados. Son los primeros elementos, según constatan las investigaciones de Alain Corbin (1987) sobre este tema, de una política de higiene pública, de una gestión higiénica de la población que tendrá que ser saneada, «protegida» de los estragos de la infección y, en particular, del "contagio moral", quedando encuadrada bajo una serie de prescripciones y proscripciones concernientes a las formas de existencia en general.

A partir de una población indigente, bulliciosa y salvaje, de vida inmunda, y como principal vector de propagación de gérmenes y miasmas, se establece un cinturón sanitario donde soltar las redes de captura higiénica. A partir de 
una atención científica y normativa del buen funcionamiento físico y moral de los cuerpos de los individuos se tratará de medicalizar todo el cuerpo social. La necesidad perentoria de la profilaxis social del higienismo reformador es la de "desinfectar" a la pobreza pero no únicamente a través de la consecución de una salud vigorosa, sino también mediante la adopción por parte de los obreros de un comportamiento moral apto para el trabajo en la fábrica, con la interiorización de las normas que los someten a las representaciones y comportamientos dominantes de la moral burguesa: sobriedad, orden, familia, ahorro.

El contraste entre las costumbres populares en materia de prácticas domésticas y privadas ligadas a la habitabilidad y las prescripciones higienistas sobre el modo de vivir en la ciudad, tiene su punto de fricción en la confluencia entre la precariedad material de una vida pobre y los valores éticos que le dan sentido como experiencia. Si todavía no se percibe una exigencia del confort, pues las circunstancias de edificabilidad todavía no lo permiten, podemos afirmar que se observan unas conquistas de lo ordinario, mediante pequeñas, ínfimas, estrategias de privacidad que se ponen a resguardo de cualquier intento de pesquisa o advertencia externa.

La presencia de malos olores, humos, aguas residuales, mezcla inmunda de desechos industriales y evacuaciones no canalizadas, no implica una indiferencia obrera en relación al entorno: un mal olor es percibido del mismo modo por un habitante de los barrios burgueses que por un trabajador de fábrica. Durante las visitas de los servicios de higiene social a los 
alojamientos obreros aperciben a sus habitantes de la necesidad de abrir las ventanas y dejar correr el aire, cuando precisamente el estar cerradas, a pesar de la insalubridad que acompaña a esta costumbre, impide que el aire viciado que circunda a los barrios obreros entre en su interior (Murard y Zylberman, 1987). Asimismo ocurre con los insectos. En verano se convierten en auténticas plagas y durante el año hay que vigilar que cualquier apicultor del barrio no haya instalado un panal de abejas cerca de la vivienda aprovechando la cercanía de alguna refinería de azúcar. Un mapa industrial de las principales ciudades europeas de principios de siglo, muestra una diseminación urbana de pequeñas fábricas y talleres cuya actividad se insemina a lo largo de una red tupida de viviendas - en el interior de un proceso de racionalización del espacio (cf. Magri y Topalov, 1989).

Finalicemos esta aproximación cultural a la vida urbana de la clase obrera con otro ejemplo que nos parece signficativo. Se trata de una disciplina a domicilio propia de una estrategia "familiarista" — en la medida en que los equipamientos colectivos acentúan de forma adyacente lo usos privados en detrimento de lo público-, a través de la progresiva instalación del agua corriente en las viviendas populares. Si bien es cierto que supone un recurso importante en la higiene de la población de las fábricas, cuyo modo de producción ensucia y contamina al obrero, también es cierto que margina formas de uso colectivo del agua reduciendo la autonomía familiar, de territorialidades colectivas tradicionales como vienen a ser los lavaderos comunales, las fuentes y las aguas subterráneas... El uso privado del agua, 
que excluye cualquier derecho subjetivo de apropiación colectiva, significará en adelante un ejercicio jurídico en manos de las administraciones, que llevarán a cabo un control sobre la distribución, el volumen y el uso, y que el obrero deberá costear por su cuenta al estar condicionada como valor de mercancía.

Barbey, G. (1990). L'évasion domestique: essai sur les relations d'affectivité au logis. Lausana: Presses Polytechniques et Universitaires Romandes.

Battagliola, F. (1995). Mariage, concubinage et relations entre les sexes. Paris, 1880-1890. Genèses, 18, 68-96.

Boucher, M. (2011). Gouverner les familles. Les classes populaires à l'épreuve de la parentalité. París: L'Harmattan.

Chevalier, L. (1984). Classes laborieuses et dangereuses à Paris pendant la premiére moitié du XIX siècle. París: Hachette.

Certeau, M. (1980). L’invention du quotidien, 1. Arts de faire. París: UGE.

Cohen, Y. y Baudouï, R. (Eds.). (1995). Les chantiers de la paix sociale: 1900-1940. Fontanay/Saint-Cloud: ENS.

Corbin, A. (1987). El perfume o el miasma. El olfato y lo imaginario social. Siglos XVIII y XIX. Madrid: FCE. 
Dean, M. (1991). The Constitution of Poverty. Towards a Genealogy of Liberal Governance. Londres: Routledge.

Dollé, J. P. (1990). Fureurs de ville. París: Grasset \& Fasquelle.

Donzelot, J. (2007). La invención de lo social: ensayos sobre la declinación de las pasiones políticas. Buenos Aires, Argentina: Nueva Visión.

Fairchild, C. (1978). Female sexual attitudes and de rise of illegitimacy: A case study. Journal of Interdisciplinary History, 8(4), 627-667.

Farge, A. y Klapisch-Zuber, C. (Eds). (1984). Madame ou Mademoiselle? Itinéraries de la solitude féminine, 18-20 siècles. París: ArthaudMontalba.

Faure, A. (1993). Le local: une approche du quartier populaire (Paris, 1880-1914). Mélanges de l'Ecole française de Rome, 105(2), 489-502.

Faure, A. (1994). Réflexions sur les ambiguités du quartier populaire (Paris, 18801914). Histoire, économie et société, 13(3), 449-455.

Faure, A. (1998) "Les couches nouvelles de la propriété". Un peuple parisien à la conquête du bon logis à la veille de la Grande Guerre. Le Mouvement Social, 182(1), 53-78.

Faure, A. (1999). Comment se logait le peuple parisien à la Belle Époque. Vingtième Siècle, 64, 41-51. 
Frioux, S., Fournier, P. y Chauveau, S. (2011). Hygiène et santé en Europe: de la fin du XVIII siècle aux lendemains de la Première Guerre Mondiale. París: SEDES.

Harloe, M. (1995). The People's Home: Social Rented Housing in Europe and America. Oxford: Basil Blackwell.

Léridon, H. y Villeneuve-Gokalp, C. (1994). Constance et inconstances de la famille: biographies familiales des couples et des enfants. París: INED.

Magri, S. (1991). Des “ouvriers” aux “citoyens modestes": Naissance d'une catégorie: les bénéficiaires des habitations à bon marché au tournant du $\mathrm{XX}^{\mathrm{e}}$ siècle. Genèses, 5, 35-53.

Magri, S. (1997). L'intérieur domestique. Pour une analyse du changement dans les manières d'habiter. Genèses, 28, 146-164.

Magri, S. y Topalov, C. (Eds.). (1989). Villes ouvrières, 1900-1950. París: L'Harmattan.

Murard, L. y Zylberman, P. (1976). Le petit travailleur infatigable ou le prolétaire régénere. Villes-usines, habitat et intimités au XIX siècle. París: CERFIRecherches.

Murard, L. y Zylberman, P. (1986). L'autre guerre, la santé publique sous l'oeil de l'Amérique. Revue historique, 560, 367-98.

Murard, L. y Zylberman, P. (1987). L'Idée de service social dans la pensée hygiéniste (1928-1936). Vie sociale, 8-9, 463-484.

Paradeise, C. (2003). French Sociology of Work and Labor: From Shop Floor to Labor Markets to Networked Careers. Organization studies, 24(4), 633-653. 
Segrestin, D. (1980). Les communautés pertinentes de l'action collective. Canevas pour l'étude des fondaments sociaux des conflits du travail. Revue française de sociologie, 2, 171-203.

Warin, Ph. (1995). Les politiques publiques, multiplicité d'arbitrages et construction de l'ordre social. En F. Alain., P. Guilles y W. Philippe (Eds.), La Construction du sens dans les politiques publiques. Débats autour de la notion de referential (pp. 85-101). París: L'Harmattan.

\section{¿Cómo citar este artículo?}

Calvo, F. (Enero-junio, 2017). Las intimidades colectivas de la Clase Obrera. Vida urbana y cultura popular en las ciudades de principios del siglo XX. Revista humanidades, 7(1), 1-24. doi: http://dx.doi.org/10.15517/h.v7i1.27622 